\title{
Robotic-assisted tracheobronchial surgery
}

\author{
Brian D. Cohen ${ }^{1}$, M. Blair Marshall ${ }^{2}$ \\ ${ }^{1}$ General Surgery Residency Program, MedStar Georgetown/Washington Hospital Center, Washington DC, USA; ${ }^{2}$ Division of Thoracic Surgery, \\ Brigham and Women's Hospital, Faculty, Harvard Medical School, Boston, MA, USA \\ Contributions: (I) Conception and design: All authors; (II) Administrative support: None; (III) Provision of study materials or patients: MB Marshall; \\ (IV) Collection and assembly of data: All authors; (V) Data analysis and interpretation: All authors; (VI) Manuscript writing: All authors; (VII) Final \\ approval of manuscript: All authors. \\ Correspondence to: Brian D. Cohen, MD. General Surgery Residency Program, MedStar Georgetown/Washington Hospital Center, Washington DC, \\ USA. Email: brian.d.cohen@medstar.net; M. Blair Marshall, MD. Associate Chief for Quality and Safety, Division of Thoracic Surgery, Brigham and \\ Women's Hospital, Faculty, Harvard Medical School, Boston, MA, USA. Email: mmarshall16@bwh.harvard.edu.
}

\begin{abstract}
Robotic technology is positioned to transform the approach to tracheobronchial surgery. With its magnified 3D view, intuitive controls, wristed-instruments, high-fidelity simulation platforms, and the steady implementation of new technical improvement, the robot is well-suited to manage the careful dissection and delicate handling of the airway in tracheobronchial surgery. This innovative technology has the potential to promote the widespread adoption of minimally invasive techniques for this complex thoracic surgery.
\end{abstract}

Keywords: Robotic surgery; thoracic surgery; sleeve lobectomy; sleeve resection; bronchoplasty

Submitted Nov 12, 2019. Accepted for publication Feb 19, 2020.

doi: $10.21037 /$ jtd.2020.03.05

View this article at: http://dx.doi.org/10.21037/jtd.2020.03.05

\section{Introduction}

Minimally invasive surgery (MIS) has revolutionized how surgeons approach a large variety of complex procedures, nowhere is this better exemplified than the robotic approach to tracheobronchial surgery.

The development of MIS techniques and the evolution of video-assisted thoracoscopic surgery (VATS) has driven novel approaches among an ever-growing population of patients amenable to MIS surgery. Previously restricted to diagnostic procedures, thoracoscopic technology can now be used to perform increasingly complex thoracic operations. Numerous retrospective studies have demonstrated advantages of these minimally invasive techniques over an open thoracotomy, citing improved post-operative pulmonary function, diminished pain, shorter length of stay, equivalent oncologic outcomes, and better overall prognosis $(1,2)$. Despite these advantages, the literature shows a sluggish adoption of MIS techniques for thoracic procedures, with over half of lobectomies still performed via open thoracotomy in 2014 (3).

As VATS procedures burgeoned, robotic systems were simultaneously being developed. Boasting its four-armed platform, 10x magnification with binocular lens for 3D vision, and wristed instruments allowing seven degrees-offreedom, the da Vinci system (Intuitive Surgical, Sunnyvale, CA, USA) has become the dominant robotic platform. Robotic-assisted thoracic surgery (RATS) pioneers swiftly accumulated surgical "firsts", including the first lobectomy, thymectomy, and esophagectomy. Proponents offer that the robot's superior visualization, improved ergonomics, more intuitive instrument movements, and ability to perform more meticulous dissection will propel RATS where surgeons have so far been slow to adopt VATS techniques (4).

The robotic approach was made even more accessible with the 2014 release of da Vinci's fourth-generation platform, Xi. With its simplified docking procedure, better maneuverability of its boom-mounted arms, and extended instrument reach, several studies have favorably compared the $\mathrm{Xi}$ to its predecessor in general surgery literature (5). For tracheobronchial surgery, we would highlight the importance of the new platform's capacity for camera "port hopping". With a redesigned, $8 \mathrm{~mm}$ endoscope, the camera can now be placed into any working port, allowing the 

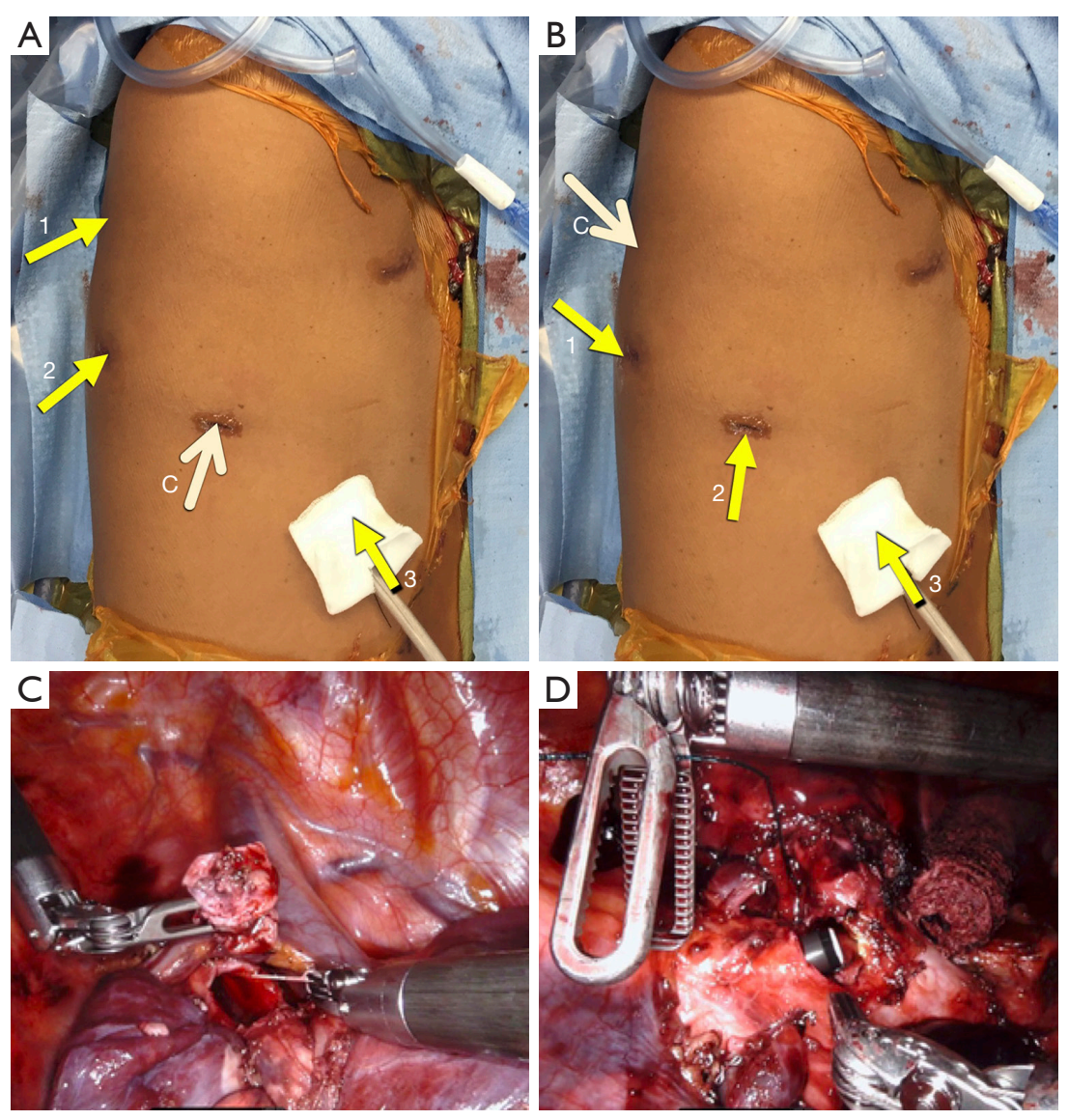

Figure 1 Robotic airway reconstruction with arrows 1-3 depicting instrument placement and arrow C denoting camera placement. (A,B) Camera placement at two different points of airway reconstruction, utilizing "port-hopping"; (C) depicts the view from (A), looking superiorly towards the apex of the chest at initial bronchotomy with lung parenchyma retracted inferiorly; (D) depicts the view from (B), looking inferior-medially over the divided azygous vein during bronchoplasty (bronchoscope can be seen in the airway lumen).

surgeon to change views without a burdensome redocking process. This is illustrated by an airway reconstruction as depicted in Figure 1. The initial camera placement seen in Figure $1 A$. provides the view obtained during dissection, bronchial resection and reconstruction (Figure 1C). The lesion resected was at the junction of the distal right mainstem bronchus and apical segmental bronchus. The camera can then be moved to the posterior-most port (Figure $1 B$ ) and bronchoplasty completed with an inferiormedial facing view (Figure 1D). This ability to "port hop" provides unparalleled opportunities for visualization during key steps of complex operations. We expect this improved visualization to facilitate the transition for more surgeons who want to incorporate MIS techniques into their approach to tracheobronchial surgery.

Robotic simulation also allows surgeons and trainees to safely practice transferrable surgical skills and serves as a tool for planning any novel procedure or approach. For example, a left upper lobe sleeve lobectomy and pulmonary arterioplasty was performed on a cadaveric porcine model (Figure 2). With the ability to simulate operations and practice technical maneuvers using its intuitive controls (via cadavers, animal models, or virtual systems), the robot promises a shorter learning curve compared to VATS. For robotic lobectomies, the learning curve among trained thoracic surgeons is roughly $20-30$ cases $(6,7)$. At this early stage, no studies exist specifically investigating tracheobronchial operations, but given the delicate dissection and current low adoption rate of MIS techniques, it is reasonable to assume the robot will outperform VATS technology.

The da Vinci system also has clear advantages when it 


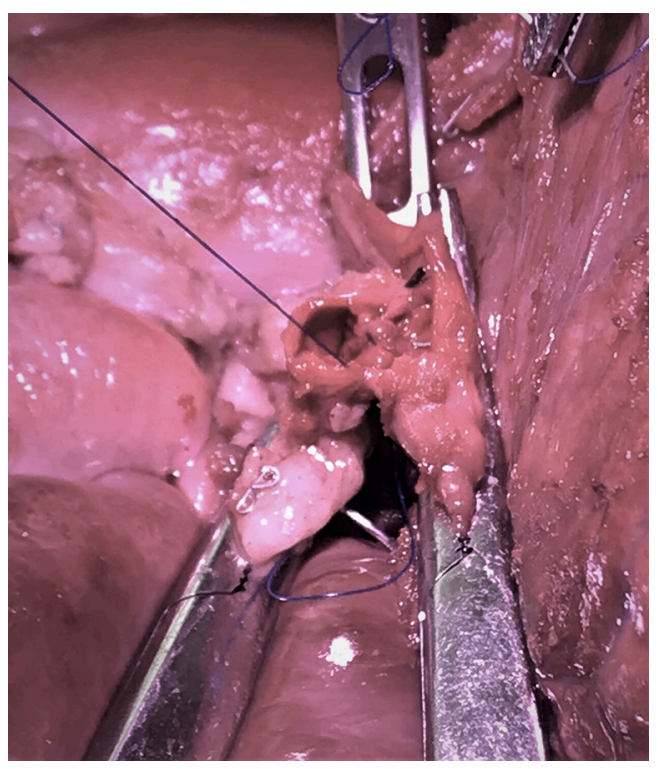

Figure 2 Pulmonary arterioplasty being performed on cadaveric porcine model.

comes to teaching tracheobronchial surgery to new trainees. Dual consoles allow an instructor to assume control of the operation from his/her trainee as needed, helping to balance safety and autonomy. This can be accomplished without switching positions or performing a technique from the opposite side of the table. The trainee and instructor have the same view and same set of "hands". In recent surveys, thoracic surgery trainees expressed the need for more instruction using the robot (8), though standardize pathways to competency have been proposed (9).

\section{Sleeve lobectomy}

Until recently, a MIS approach has been contraindicated for sleeve lobectomies due to technical difficulty and concerns for achieving a complete oncologic resection. However, as studies have reinforced the superiority of sleeve lobectomy to pneumonectomy (10), surgeons at experienced centers have demonstrated success using MIS techniques (11-13).

In their series of 15 patients undergoing MIS sleeve resections, including two with robotic approaches, Caso et al. demonstrated outcomes comparable to open thoracotomies (14), adding evidence that the MIS approach is feasible with acceptable morbidity. Two robotic cases described were a complete portal robotic distal tracheal and left main stem resection and reconstruction on extracorporeal membrane oxygenation (ECMO) for granular cell tumor and a bronchus intermedius resection for bronchial adenoma. For both cases, the authors described a four-port technique using the da Vinci Xi system. Notably, while preparing for the distal tracheal and left main stem resection and reconstruction, the operating surgeons utilized a cadaveric model to plan the novel approach (15). Though an MIS approach was not initially thought to be possible, the surgeons reconsidered given growing experience using the robotic approach. By first practicing the technique on a cadaver, they demonstrated its feasibility and reduced any propensity for technical errors. The definitive operation was performed a week later with no major complications, the patient was discharged home on post-operative day (POD) 5. The second patient undergoing the robotic approach in their MIS series was discharged on POD 6 without major complications. Both patients demonstrated no evidence of disease or any notable complications at follow-up after 2 and 7 months, respectively.

These results are consistent with a series of eight patients undergoing a robotic sleeve lobectomy written by Cerfolio (16). In this series, he details the robotic setup and operative technique for a right upper lobe sleeve lobectomy using the da Vinci Si system. Of their eight patients, one required conversion to thoracotomy after injury to the apical segment of the pulmonary artery caused bleeding. No blood transfusion was required, and the patient did well after an open sleeve lobectomy. All patients tolerated the operation well, with no major post-operative morbidity or mortality. Mean length of stay was 3.1 \pm 0.9 days. Egberts et al. describe a similar robotic technique, detailing small modifications for the da Vinci Xi system (17).

In total, early case series demonstrate the promise of a robotic technique for these difficult thoracic operations. VATS sleeves are only being performed in a small handful of centers pushing the boundaries of MIS techniques. While the RATS approach is still limited to programs with a robust robotic program, the new technology has the potential for wider adoption. With the delicate nature of an airway anastomosis, the improved vision, more precise dissection, and high-fidelity simulation made possible by the robot, this new technology may allow surgeons to more easily transition from traditional thoracotomy to MIS. In this current period of early adoption and novel techniques, future studies are still needed to compare outcomes and adoption rates between robotic, open, and VATS approaches for sleeve lobectomies. 


\section{Tracheobronchial malacia (TBM)}

Patients with TBM represent another population that may benefit from a novel robotic approach to an operation that has traditionally only been offered via open thoracotomy. Characterized by a weakness and collapse of the trachea and/or mainstem bronchi, TBM is often acquired. It afflicts those with pre-existing respiratory disease, most commonly asthma with chronic inhaled-steroid use or chronic pulmonary obstructive disorder (COPD), and is often underdiagnosed and undertreated. Temporary treatment options include airway stenting, either with silicone Y-stents or expandable metal stents. These may cause foreign-body inflammation, mucus plugging, infection, or a difficult retrieval process, and are only indicated as a short-term solution (18). The definitive treatment remains open tracheobronchoplasty via right posterolateral thoracotomy to plicate redundant membrane and stabilize the airway with mesh placement. This approach, however, carries the expected drawbacks of open thoracotomy in this morbid patient population and has mixed results in improved forced expiratory volume at 1 second (FEV1), symptom relief, and improved quality of life (19).

Lazzaro et al. described a series of 42 patients undergoing robot-assisted tracheobronchoplasty for TBM (20) with the goal of demonstrating the safety and improved outcomes using a robotic technique. The authors describe a 4-port approach (plus one 12-mm assistant port) using the da Vinci $\mathrm{Xi}$ platform to stent the airway by secure polypropylene braided mesh. In their population, the median length of stay was 3-day and median ICU length of stay was 1 day. They described 19 post-operative complications, 11 minor (26\%) and 8 majors (19\%). The major complications included, two chest tubes required for pneumothoraces, symptomatic subcutaneous emphysema, one hemothorax requiring intervention, and two patients needing to return to the ICU (for bronchoscopy and monitoring of an arrhythmia). One patient also required a revision surgery for persistent symptoms at 125-day. There were no 90-day mortalities and no reintubations or tracheostomies. Pulmonary function testing (PFT) showed statistically significant improvement in FEV1, forced vital capacity, and peak expiratory flow rate, by $13.5 \%(\mathrm{P}=0.01), 14.5 \%(\mathrm{P}<0.0001)$, and $21.0 \%$ $(\mathrm{P}<0.0001)$, respectively. They also demonstrated an $82 \%$ improvement on quality of life questionnaire.

This demonstrated safety and improved PFTs after robotic-tracheobronchoplasty for TBM offer a promising option for this historically under-treated disease process.
The robotic platform may allow more surgeons to offer definitive treatment to this difficult patient population.

\section{Disadvantages}

Despite the advantages previously described, the robotic approach to tracheobronchial surgery carries costs. Existing literature points out the greater expense and longer operating times compared to VATS (21), while many have noted the lack of tactile feedback, high upfront logistic considerations for hospitals without robotic programs, and the lack of standardized credentialing or training programs (22). Though common for new technologies, and sure to improve as the field matures, these costs and lessons learned from the robotic lobectomy experience (23) will likely apply equally to tracheobronchial procedures.

Additionally, robotic tracheobronchial surgery requires particular attention from an experienced anesthesiology team, with specific expertise managing $\mathrm{CO}_{2}$ insufflation, capnothorax, single-lung ventilation, and the potential need for ECMO $(24,25)$. As always, clear communication between surgical and anesthesia teams is essential to ensure patient safety, a successful operation, and adequate postoperative pain control.

\section{Conclusions}

With great technologic advancements, the approach to tracheobronchial surgery is rapidly being transformed. Robotic surgery can promote the widespread adoption of MIS techniques for complex thoracic surgery, succeeding where VATS has been slow to achieve application more broadly. Its magnified 3D view, intuitive controls, wristedinstruments, high-fidelity simulation platforms, and the steady implementation of new technical improvement leave the robot well-suited to manage the careful dissection and delicate handling of the airway in tracheobronchial surgery.

We look forward to future studies comparing rates of adoption, short-term outcomes, and long-term benefits between robotic surgery, VATS, and open thoracotomy. As more surgeons gain familiarity with the robotic approach to tracheobronchial surgery, we anticipate that more patients will benefit from these innovative tools.

\section{Acknowledgments}

Funding: None. 


\section{Footnote}

Provenance and Peer review: This article was commissioned by the Guest Editor (Servet Bölükbas) for the series "Airway Surgery", published in Fournal of Thoracic Disease. This article has undergone external peer review.

Conflicts of Interest: Both authors have completed the ICMJE uniform disclosure form (available at http://dx.doi. org/10.21037/jtd.2020.03.05). The series "Airway Surgery" was commissioned by the editorial office without any funding or sponsorship. The authors have no other conflicts of interest to declare.

Ethical Statement: The authors are accountable for all aspects of the work in ensuring that questions related to the accuracy or integrity of any part of the work are appropriately investigated and resolved.

Open Access Statement: This is an Open Access article distributed in accordance with the Creative Commons Attribution-NonCommercial-NoDerivs 4.0 International License (CC BY-NC-ND 4.0), which permits the noncommercial replication and distribution of the article with the strict proviso that no changes or edits are made and the original work is properly cited (including links to both the formal publication through the relevant DOI and the license). See: https://creativecommons.org/licenses/by-nc-nd/4.0/.

\section{References}

1. Kaseda S, Aoki T, Hangai N, et al. Better pulmonary function and prognosis with video-assisted thoracic surgery than with thoracotomy. Ann Thorac Surg 2000;70:1644-6.

2. Bendixen M, Jørgensen OD, Kronborg C, et al. Postoperative pain and quality of life after lobectomy via video-assisted thoracoscopic surgery or anterolateral thoracotomy for early stage lung cancer: a randomised controlled trial. Lancet Oncol 2016;17:836-44.

3. Blasberg JD, Seder CW, Leverson G, et al. Video-assisted thoracoscopic lobectomy for lung cancer: current practice patterns and predictors of adoption. Ann Thorac Surg 2016;102:1854-62.

4. Kumar A, Asaf BB. Robotic thoracic surgery: the state of the art. J Minim Access Surg 2015;11:60-7.

5. Ngu JC, Tsang CB, Koh DC. The da Vinci Xi: a review of its capabilities, versatility, and potential role in robotic colorectal surgery. Robot Surg 2017;4:77-85.

6. Melfi FM, Mussi A. Robotically assisted lobectomy: learning curve and complications. Thorac Surg Clin 2008;18:289-95, vi-vii.

7. Song G, Sun X, Miao S, et al. Learning curve for robot-assisted lobectomy of lung cancer. J Thorac Dis 2019;11:2431-7 .

8. Chu D, Vaporciyan AA, Iannettoni MD, et al. Are there gaps in current thoracic surgery residency training programs? Ann Thorac Surg 2016;101:2350-5.

9. Cerfolio RJ, Bryant AS. How to teach robotic pulmonary resection. Semin Thorac Cardiovasc Surg 2013;25:76-82.

10. Stallard J, Loberg A, Dunning J, et al. Is a sleeve lobectomy significantly better than a pneumonectomy? Interact Cardiovasc Thorac Surg 2010;11:660-6.

11. Bertolaccini L, Viti A, Terzi A. Moving beyond the boundary: the emerging role of video-assisted thoracic surgery for bronchoplastic resections. J Thorac Dis 2014;6:1170-2.

12. Huang J, Li J, Qiu Y, et al. Thoracoscopic double sleeve lobectomy in 13 patients: a series report from multicenters. J Thorac Dis 2015;7:834-42.

13. Gonzalez-Rivas D, Yang Y, Sekhniaidze D, et al. Uniportal video-assisted thoracoscopic bronchoplastic and carinal sleeve procedures. J Thorac Dis 2016;8:S210-22.

14. Caso R, Watson TJ, Khaitan PG, et al. Outcomes of minimally invasive sleeve resection. J Thorac Dis 2018;10:6653-9.

15. Caso R, Khaitan PG, Shults CC, et al. Simulation for Technical Challenge: Complete Portal Robotic Distal Tracheal and Left Main Stem Resection and Reconstruction on ECMO. CTSNet 2018. doi:10.25373/ctsnet.7388969.

16. Cerfolio RJ. Robotic sleeve lobectomy: technical details and early results. J Thorac Dis 2016;8:S223-6.

17. Egberts JH, Möller T, Becker T. Robotic-assisted sleeve lobectomy using the four-arm technique in the $\mathrm{DaVinci} \mathrm{Si}{ }^{\circledR}$ and $\mathrm{Xi}{ }^{\circledR}$ Systems. Thorac Cardiovasc Surg 2019;67:603-5.

18. Gangadharan SP. Tracheobronchomalacia in adults. Semin Thorac Cardiovasc Surg 2010;22:165-73.

19. Gangadharan SP, Bakhos CT, Majid A, et al. Technical aspects and outcomes of tracheobronchoplasty for severe tracheobronchomalacia. Ann Thorac Surg 2011;91:157480; discussion 1580-1.

20. Lazzaro R, Patton B, Lee P, et al. First series of minimally invasive, robot-assisted tracheobronchoplasty with mesh 
for severe tracheobronchomalacia. J Thorac Cardiovasc Surg 2019;157:791-800.

21. Augustin F, Bodner J, Maier H, et al. Robotic-assisted minimally invasive vs. thoracoscopic lung lobectomy: comparison of perioperative results in a learning curve setting. Langenbecks Arch Surg 2013;398:895-901.

22. Cerfolio RJ. Pulmonary resection in the 21 st century: the role of robotics. Tex Heart Inst J 2012;39:848-9.

23. Cerfolio RJ. Total port approach for robotic lobectomy. Thorac Surg Clin 2014;24:151-6, v.

24. Zhang Y, Wang S, Sun Y. Anesthesia of robotic thoracic surgery. Ann Transl Med 2015;3:71.

25. Chitilian HV, Bao X, Mathisen DJ, et al. Anesthesia for airway surgery. Thorac Surg Clin 2018;28:249-55.
Cite this article as: Cohen BD, Marshall MB. Robotic-assisted tracheobronchial surgery. J Thorac Dis 2020;12(10):6173-6178. doi: $10.21037 /$ jtd.2020.03.05 